\title{
Selection of Feedback Signals for Controlling Dynamics in Future Power Transmission Networks
}

\author{
Linash P. Kunjumuhammed, Member, IEEE, and Bikash C. Pal, Fellow, IEEE
}

\begin{abstract}
This paper deals with the selection of feedback signal(s) that retain the modal behavior of power system electromechanical dynamics under varying operating circumstances. The approach seeks signals that have relatively large magnitude of residue, less variation of the magnitude and phase angle, sufficient gap between the critical pole-zeros, and least sensitive to other modes. The methodology is tested in a 16-machine interconnected power system model with multiple wind farms.
\end{abstract}

Index Terms-Damping, interarea oscillation, signal selection, small signal stability.

\section{INTRODUCTION}

$\mathbf{T}$ HE STABILITY of interconnected power system under widely varying operating scenarios has always been a challenge. In view of increasing generation from the renewables, the operation of the system is going to be far more challenging. Traditionally, the electromechanical dynamics in power system have been controlled through power system stabilizer (PSS) and flexible ac transmission system devicesbased power oscillation damping (POD) controllers [1]. Flexible alternating current transmission systems (FACTS) devices have been installed in specific locations of the network mainly from the consideration of voltage support and power-flow control. The number of FACTS controllers is growing to alleviate congestion in critical power transfer path. The congestion is primarily anticipated to transport the power from renewable rich area in the system. It has also been reported that stability and control contribution from the wind farm to the overall interconnected dynamic response of the system is also potentially attractive [2].

The selection of feedback control signal so far has been dominated by the modal observability of the signal. In view of more FACTS devices in the system and also the deployment of phasor measurement unit technology into the network, there is an opportunity to choose control device and feedback signal that produce larger modal controllability and observability. Given the widely varying operating scenarios-the selection of effective signal is now far more difficult requiring

Manuscript received March 24, 2014; revised August 4, 2014; accepted October 9, 2014. Paper no. TSG-00265-2014. (Corresponding Author: Bikash C. Pal.)

The authors are with the Department of Electrical and Electronic Engineering, Imperial College London, London SW7 2AZ, U.K. (e-mail: b.pal@imperial.ac.uk).

Color versions of one or more of the figures in this paper are available online at http://ieeexplore.ieee.org.

Digital Object Identifier 10.1109/TSG.2014.2362967 a very systematic and robust approach. This paper proposes a procedure to select feedback signal for PSS and POD controllers considering large number of operating conditions. The proposed approach simplifies controller design and ensures robust damping performance over a widely varying operating conditions.

This paper is organized as follows. Various methods proposed in the literature for signal selection is discussed in Section II. Following main concepts from the discussion, a set of signal selection criteria and an algorithm for signal selection are presented in Section III. The method is validated through simulation results performed on a 16-machine test system with about $12 \%$ of the total generation from the wind. The test system and various operating conditions are described in Section IV. Section V provides results of modal analysis under all selected operating conditions. The effectiveness of the control through the selected signal is demonstrated in Section VI.

\section{Signal Selection Methods}

The dynamics of power system in linearized form is represented as

$$
\dot{\mathbf{x}}=\mathbf{A x}+\mathbf{B u}, \quad \mathbf{y}=\mathbf{C x}+\mathbf{D u} .
$$

A, B, C are state, input, and output matrices respectively. $\mathrm{D}$ directly connects input (u) with output (y). The electromechanical modes are obtained from some complex eigenvalues of $\mathrm{A}\left\{\lambda_{i}=\sigma_{i} \pm j \omega_{i}\right\}_{1}^{n} \cdot f_{i}=\omega_{i} / 2 \pi$ and $\zeta_{i}=-\sigma_{i} / \sqrt{\sigma_{i}^{2}+\omega_{i}^{2}}$ represent frequency in $\mathrm{Hz}$ and damping ratio of the $i$ th mode, respectively. The transfer function representation of (1) between $j$ th input and $k$ th output is written as

$$
\mathbf{G}(\mathbf{s})=\sum_{i=1}^{i=n} \frac{R_{i}}{s-\lambda_{i}}=\frac{k\left(s-z_{1}\right)\left(s-z_{2}\right) \ldots\left(s-z_{m}\right)}{\left(s-p_{1}\right)\left(s-p_{2}\right) \ldots\left(s-p_{n}\right)}
$$

where $R_{i}=\mathbf{C}_{\mathbf{k}} \phi_{\mathbf{i}} \psi_{\mathbf{i}} \mathbf{B}_{\mathbf{j}}$ is modal residue corresponding to $\lambda_{i}$. Symbols $\phi_{i}$ and $\psi_{i}$ are right and left eigenvectors corresponding to $\lambda_{i}$, respectively. Also, $p_{i}$ and $z_{i}$ are poles and zeros of the transfer function, respectively.

The residue is a complex variable with magnitude $\left(\left|R_{i}\right|\right)$ and angle $\left(\angle R_{i}\right)$. Suppose a controller is designed with transfer function $K H(s)$ to improve the damping of $i$ th mode $\lambda_{i}$, where $K$ is the controller gain and the angle $\angle H\left(\lambda_{i}\right)=-\angle R_{i}$. The sensitivity of the eigenvalue to the gain is given by [3], [4]

$$
\Delta \lambda_{i}=\Delta K R_{i} H\left(\lambda_{i}\right) .
$$

1949-3053 (c) 2014 IEEE. Translations and content mining are permitted for academic research only. Personal use is permitted, but republication/ redistribution requires IEEE permission. See http://www.ieee.org/publications_standards/publications/rights/index.html for more information. 
Equation (3) implies that a signal-actuator pair with largest $\left|R_{i}\right|$ is the best to provide required eigenvalue shift with least control effort. Many literatures have used this concept to select location of actuator and feedback signal [1], [3]-[8]. However, as the operating condition changes, the residue varies. Wang [9] proposes two additional constraints for signal selection. For $m$ operating conditions and $n$ potential signal-actuator combinations, the selection is based on the following.

1) $\max _{n}\left[\min _{m}\left|R_{i}(n, m)\right|\right]$, which means the most effective combination is selected at the operating condition where the stabilizer is least effective.

2) $\min _{n}\left[\max _{m}\left|R_{i}(n, m)\right|-\min _{m}\left|R_{i}(n, m)\right|\right]$, such that the variation in damping contribution is minimum across all considered operating conditions.

Fan and Feliachi [10] further extended the work in [9] to obtain the selection such that the sensitivity of the controller to closely located modes are very small. This is ensured by selecting signals having less variation in $|R|$ corresponding to those modes. Kunjumuhammed et al. [11] and [12] demonstrated that by choosing signals with least variation in magnitude and angle of residue adequate damping could be provided to interarea oscillation modes. Fan and Feliachi [13] and Ray et al. [14] put forward a constraint that the residue phase of oscillation modes at close frequencies are to be bundled together within narrow angle $\left(<90^{\circ}\right)$. This is because of the fact that the required phase angle variation is difficult to achieve within a narrow frequency range using low-order controllers and closeness in angles of the residue will shield other modes from negative influence.

While the residues indicate the movement of eigenvalues (poles) for small gain, the large gain behavior is determined by location of the zeros. A good separation between the critical poles and zeros is required in order to ensure a required eigenvalue movement toward left half of eigen-plane. Reference [15] addresses the issue of zeros in the selection of actuator-signal combination for damping controller design. A suitable zero placement can be achieved by modifying the local signals [15] or changing the dynamics of other parts of the system [16]-[18]. A combination of minimum singular value, right half-plane zeros, relative gain array, and Hankel singular values are proposed in [19] to determine the best signal for a single input single output (SISO) and multiple input multiple output (MIMO) controller.

A geometric interpretation of the residue is used in many literature to select the feedback signals for damping controller design [20], [21]. A joint modal controllabilityobservability measure is defined as, $m_{\text {coi }}=m_{c i} m_{o i}$, where $m_{c i}=\cos \left(\angle\left(\psi_{i}, b\right)\right)$ and $m_{o i}=\cos \left(\angle\left(\phi_{i}, c\right)\right)$. The $m_{c i}$ is modal controllability measure which is equal to the cosine of the acute angle between $\psi$ and input vector $b ; m_{o i}$ is modal observability measure which is equal to the cosine of the acute angle between $\phi$ and output vector $c$.

The methods discussed so far are shown to provide good results considering few critical operating conditions. However, the difficulty arises when the operating conditions become dependent on nondispatchable generation such as wind and solar, and the number of potential signal-actuator pair to pick from are several. In the next section, a signal selection approach is developed considering large variation in operating conditions.

\section{Approach of Signal SElection}

Based on the discussions so far, an effective feedback signal must meet the following criteria.

$\mathrm{C} 1$ : The best signal-actuator combination is the one having highest $\left|R_{i}\right|$ for all operating conditions.

C2: In order to have a uniform damping contribution, the variation in $\left|R_{i}\right|$ and $\angle R_{i}$ should be minimum.

C3: The sensitivity of the controller to closely situated modes should be positive or if negative, it should be appreciably small.

C4: A good separation is required between the critical poles and zeros to ensure adequate eigenvalue movement.

Let us assume that a potential actuator is selected or decided a priori, the objective is to find the suitable feedback signal(s). Let $\left|R_{i}^{m k}\right|$ and $\theta_{i}^{m k}$ be the magnitude and angle of the residue with respect to $m$ th operating scenario corresponding to the $k$ th signal, respectively, of the $i$ th mode. The following three criteria are formulated based on the attributes already described.

\section{A. Criteria-1: Control Effectiveness}

Criteria-1 is formulated to satisfy residue characteristics $\mathrm{C} 1$. As the operating condition of the system changes, the residue magnitude changes. For a large number of operating conditions, the average magnitude of the residue $\overline{\left|R_{i}^{k}\right|}=$ $1 / N \sum_{m=1}^{N}\left|R_{i}^{m k}\right|$ is a good indicator of control effectiveness. The signals having $\overline{\left|R_{i}^{k}\right|}>L m t_{1}$ can be collected for further analysis where $L m t_{1}$ will be decided by the designer. In this paper, the signals within top $70 \%$ in order of decreasing magnitude are selected. However, the usage of average value over a large number of operating conditions should be carried out with caution as a higher magnitude of the residue for some operating conditions cannot compensate for very low value of residue for other operating conditions. This can be ensured by selecting a signal with less variation in magnitude of the residue. Criteria-2 presented below automatically takes care of this factor.

\section{B. Criteria-2: Robustness Aspect}

This criteria is developed to comply with the attribute C2 to ensure a robust performance under all operating conditions. The essence of the criteria is to find a signal with least variation in magnitude and angle of the residue under all operating conditions [11], [12]. For this, a concept of relative residue error covariance matrix $\left(\mathbf{P}_{i}^{\mathrm{rel}, k}\right)$ is used. In theory, the variation in the residue can be related to the size of $\left(\mathbf{P}_{i}^{\mathrm{rel}, k}\right)$ [22]. $\left(\mathbf{P}_{i}^{\mathrm{rel}, k}\right)$ is computed for each signal $(k)$ over a $N$ operating conditions. For a given signal, $\mathbf{P}_{i}^{\text {rel, } k}$ can be represented by an ellipse in 2-D plane [22]. The smaller the area of the error ellipse, lesser is the variation in corresponding residue. Area $\left(\mathbf{P}_{i}^{\text {rel }, k}\right) \propto$ $\pi \sqrt{\operatorname{det}\left(\mathbf{P}_{i}^{\text {rel, } k}\right)}$; thus in principle, the signal having a least value of $\operatorname{det}\left(\mathbf{P}_{i}^{\mathrm{rel}, k}\right)$ is the one having least variation in the residue. 
TABLE I

Criteria 3: Influence of NeAR-BY-Mode on Signal Selection

\begin{tabular}{l|l|l|c}
\hline Case & Angle criteria & Magnitude criteria & Remark \\
\hline $\mathrm{A}$ & $\overline{\angle R_{a}^{k}}-\overline{\angle R_{b}^{k}}<90^{0}$ & $\overline{\left|R_{a}^{k}\right|}>L m t_{1}$ & pass \\
$\mathrm{B}$ & $\frac{\angle R_{a}^{k}}{\angle R_{b}^{k}}<90^{0}$ & $\frac{\left|R_{a}^{k}\right|}{\left|R_{b}^{k}\right|}>L m t_{1}$ & pass \\
$\mathrm{C}$ & $\frac{\angle R_{a}^{k}}{\angle R_{b}^{k}}>90^{0}$ & $\frac{\left|R_{b}^{k}\right|}{|| R_{a}^{k} \mid}>L m t_{2}$ & fail \\
$\mathrm{D}$ & $\frac{\angle R_{a}^{k}}{\angle R_{b}^{k}}>90^{0}$ & $\frac{\left|R_{b}^{k}\right|}{|| R_{a}^{k} \mid}<L m t_{2}$ & pass \\
\hline
\end{tabular}

\section{Criteria-3: Reduction of Influence on Closely Situated Modes}

Often large power systems have more than one electromechanical modes which are located very close to each other. Since electromechanical modes fall in a narrow frequency range, typically less than $2 \mathrm{~Hz}$, it is difficult to design a controller for one mode while minimizing its impact on the other. This is the case when magnitude of the residues corresponding to the modes are high. The actual impact will depend upon the angle of the residue corresponding to each mode. If all of the modes located in close proximity have residue angles within a narrow range, they will be shifted almost in the same direction and vice versa. The feedback signal must be selected such that, a signal selected for one mode does not contribute to negative damping to the other by moving that modes toward the right half of the eigen-plane. This is particularly important when designing multiinput controllers where each signal is selected to influence one or more mode and therefore, two signals should not be having opposing damping contribution to a particular mode. This criteria is developed to reduce such interactions.

It is summarized in Table I. Consider a power system with two closely located electromechanical modes, mode-a and mode-b. The objective is to find a feedback signal to improve the damping of mode-a. $\overline{\left|R_{a}^{k}\right|}$ and $\overline{\left|R_{b}^{k}\right|}$ are average magnitude of the residue corresponding to signal $k$ for mode-a and mode-b, respectively. Similarly, $\overline{\angle R_{a}^{k}}$ and $\overline{\angle R_{b}^{k}}$ represent average angle of the residues corresponding to signal $k$ for mode-a and mode-b, respectively. The following four cases are possible. The cases are valid when variation in the residue i.e., $\operatorname{det}\left(\mathbf{P}^{\text {rel, } \mathbf{k}}\right)$ is small.

Case- $A$ : $\overline{\angle R_{a}^{k}}-\overline{\angle R_{b}^{k}}<90^{\circ}$ and $\overline{\left|R_{a}^{k}\right|}>L m t_{1}$ : This case ensures that the angle of the residue for both the modes are in the same angle quadrant and the signal is effective to mode-a. A fixed parameter controller designed for mode-a will not cause poor damping to mode-b, as it will also shift the mode with additional positive damping. The signal can be selected to improve the damping of mode-a.

Case- $B: \overline{\angle R_{a}^{k}}-\overline{\angle R_{b}^{k}}<90^{\circ}$ and $\overline{\left|R_{a}^{k}\right|}, \overline{\left|R_{b}^{k}\right|}>L m t_{1}$ : This is special case of Case-A, where the signal is effective for both the modes. In this case, one signal is enough to damp both the modes, if required. For better results, $\overline{\angle R_{a}^{k}}$ and $\overline{\angle R_{b}^{k}}$ must be within narrow range with less variation of the angle of the residue.

Case-C: $\overline{\angle R_{a}^{k}}-\overline{\angle R_{b}^{k}}>90^{\circ}$ and $\overline{\left|R_{b}^{k}\right|} / \overline{\left|R_{a}^{k}\right|}>L m t_{2}$ : In this case, $L m t_{2}$ is chosen based on the designers' judgment. In this paper, $L m t_{2}=0.33 . \overline{\angle R_{a}^{k}}-\overline{\angle R_{b}^{k}}=>90^{\circ}$ means that the angle

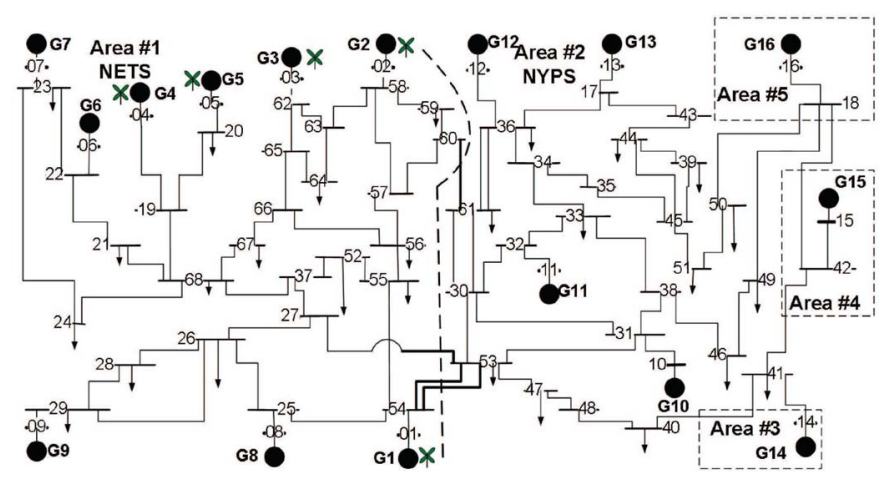

Fig. 1. Sixteen machine five-area test system.

of the residue for both the modes are not in the same quadrant. Also, $\overline{\left|R_{b}^{k}\right|} / \overline{\left|R_{a}^{k}\right|}>L m t_{2}$ suggests that the signal is effective for both of the modes. A fixed parameter controller improving the damping of mode-a is likely to reduce the damping of mode-b. Hence, such signals should not be selected.

Case-D: $\overline{\angle R_{a}^{k}}-\overline{\angle R_{b}^{k}}>90^{\circ}$ and $\overline{\left|R_{b}^{k}\right|} / \overline{\left|R_{a}^{k}\right|}<L m t_{2}$ : In this case, the angle of the residue for both the modes are not in the same quadrant. However, the control impact of the signal to mode-b is small compared to mode-a. A fixed parameter controller improving the damping of mode-a cannot significantly reduce the damping of mode-b. Hence, this signal can be used to improve the damping of mode-a.

If all other conditions are satisfied and the variation in the residue is not small, the designer's judgment based on the magnitude and angle of the residue is important. For example, a signal falls under Case-A but the variation in the residue of mode-b is high. The signal can be selected, if the control impact of that signal to mode-b is appreciably small.

A large number of potential feedback signals can be selected for a given actuator to start with signal selection. But this will make the modal analysis for large number of operating conditions a cumbersome task. A designer can narrow down the locations of the potential feedback signals: 1) to areas close to the participating generator in the case of a local mode; and 2 ) in the corridor between participating generators in case of interarea mode, as the corresponding $|R|$ will be higher in signals in these areas. The designer's judgment is also important for application of the criteria presented. While all the characteristics are very important, appropriate priorities has to be decided by the designer through choice of $L m t_{1}$ and $L m t_{2}$.

\section{Test System and Operating Conditions}

The proposed signal selection procedure is validated through simulation studies using a 16-machine, five-area test system model shown in Fig. 1. It is a modified version of NETS-NYPS model [23]. The first five synchronous generators, machines G1-G5, in the original system are replaced by wind farm of similar capacity. A thyristor controlled series capacitor (TCSC) is present in the line connecting buses \#18 and \#50 with 50\% compensation under nominal operating condition.

\section{A. Operating Conditions}

The selection of an effective feedback signal that ensures robust damping control depends on the set of operating 


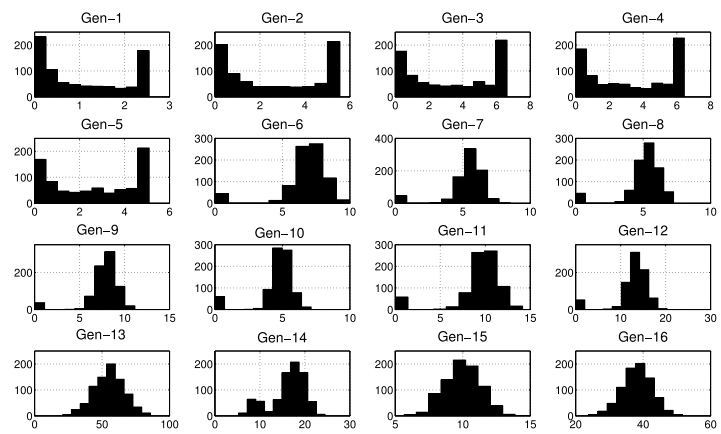

Fig. 2. Histogram showing variation in generation for different operating conditions.

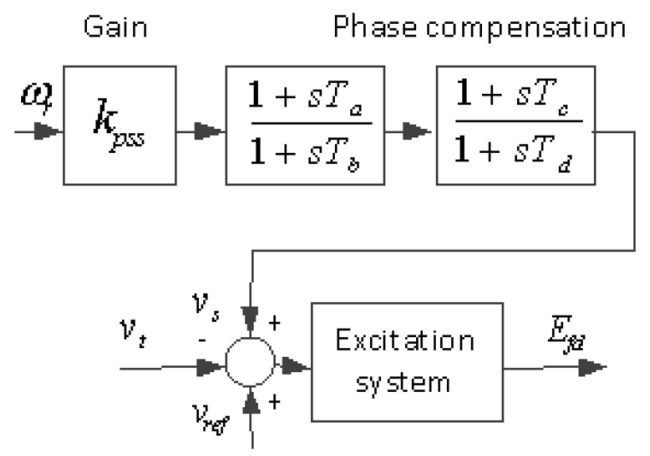

Fig. 3. Small signal dynamic model of PSS.

conditions used. It is important to generate the set which can adequately describe all possible scenarios in the power system. We have produced 800 operating scenarios in which 300 are generated without contingency. For all operating conditions, synchronous generator output, load, and wind velocity are varied.

1) Output of Synchronous Generator and Load: The uncertainty associated with both synchronous generator outputs and loads are modeled as Gaussian distribution [24]. The nominal operating condition (generation and load) is taken to be the mean $\left(\mu_{i}\right)$ of this distribution and the other operating scenarios are generated by considering a certain percentage of uncertainty around the mean. The standard deviation of the distribution $\sigma_{i}=\mu_{i} \times \%$ uncertainty $/(3 \times 100)$, where $\mathcal{N}(0,1)$ is standard normal distribution with zero mean and unit variance. In this paper, the uncertainty in synchronous generation and load (\%uncertainty) is considered to be $40 \%$. However, this value is system specific.

2) Output of Wind Generator: Unlike synchronous generators, a wind farm output is the sum of several wind turbine outputs. The output is not demand driven but depends on the availability of wind. The Weibull distribution is used for the wind generator output that reflects annual wind speed distribution for many sites [25], [26].

3) Contingency: Generator contingencies are applied on generators G6-G16, except G13 which is a slack generator. Fifty operating conditions are simulated for each contingency. For contingency on generators G14-G16, the generator output is reduced to $50 \%$ of nominal value representing collective generation of an area. The wind generator contingency is not considered separately, as its output will become zero during

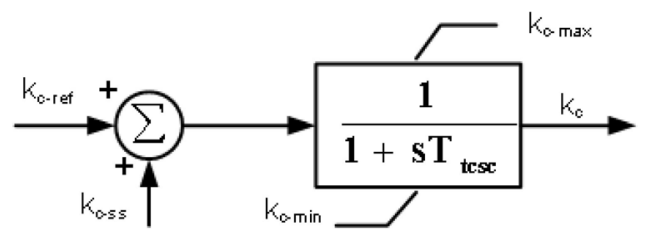

Fig. 4. Small signal dynamic model of TCSC.

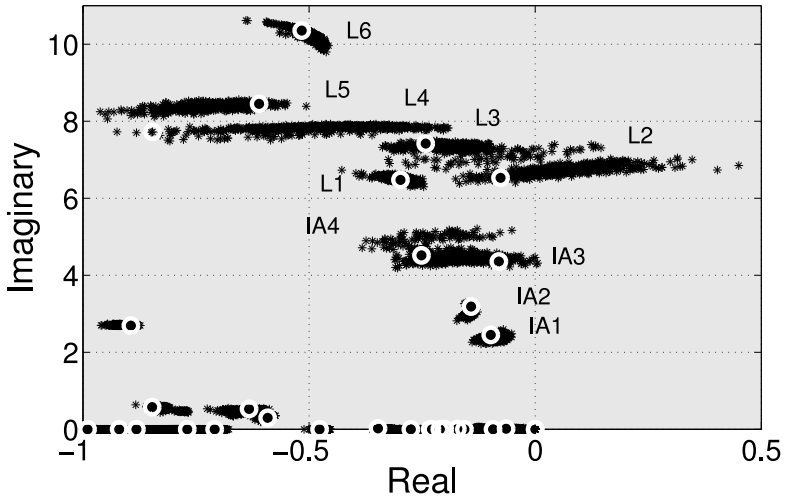

Fig. 5. Electromechanical modes of the 16-machine system for all the operating conditions. The modes at nominal operating condition is highlighted.

low wind speed periods. For each of the cases, the power-flow analysis is carried out.

1) Bus voltages are within 0.95 p.u. and 1.05 p.u.

2) Wind generator power factor is within 0.95 and unity.

3) Power factor of synchronous generators ranges from 0.9 lag and 0.85 lead.

4) Slack bus generation is $<1.2$ times its nominal generation.

The limits are imposed to generate reasonably realistic operating conditions of the system.

We believe 800 cases representing a wide range of operating conditions of the system are adequate to demonstrate the proposed signal selection method. In practice, further possible changes of power system such as line outages must be explored to obtain more possible operating conditions of the system.

\section{B. Scenario Representation}

Fig. 2 is histograms of the outputs from all 16 generation sites. The two peaks in Gen-1-Gen-5 represent zero output and rated output of wind generators. The histograms Gen-6-Gen-16 show normal distribution except a peak at zero which represents generator contingency.

\section{Detailed Modal Analysis of the System}

The test system is modeled in MATLAB/Simulink platform using transient model for synchronous machine and generic Type-3 model for wind farms. Fig. 3 shows block diagram representation of PSS. A simplified model of TCSC [23] shown in Fig. 4 is used where $k_{c}=X_{C} / X_{L}$ represents percentage compensation. The loads are assumed as static. The state space representation and the eigenvalues of the system are obtained using MATLAB commands linmod and eig, respectively.

The oscillatory modes of the system in the local and interarea mode frequency range are plotted in Fig. 5. The 
TABLE II

CRitical Eigenvalue And Dominant States of 16-Machine System AT NOMinal OpERATING CONDITION

\begin{tabular}{c|c|c}
\hline Mode & Eigenvalue & Dominant states \\
L6 & $-0.51 \pm 10.3 i$ & $\omega, \delta$ of G11 \\
L5 & $-0.61 \pm 8.4 i$ & $\omega, \delta$ of G7, G6 \\
L4 & $-0.84 \pm 7.7 i$ & $\omega, \delta$ of G8, G9 \\
L3 & $-0.24 \pm 7.4 i$ & $\omega, \delta$ of G10 \\
L2 & $-0.07 \pm 6.5 i$ & $\omega, \delta$ of G9, G6, G7, G8 \\
L1 & $-0.29 \pm 6.4 i$ & $\omega, \delta$ of G12, G13 \\
IA4 & $-0.08 \pm 4.3 i$ & $\omega, \delta$ of G6, G7,G8, G9, G13 \\
IA3 & $-0.25 \pm 4.5 i$ & $\omega, \delta$ of G14, G15,G16 \\
IA2 & $-0.14 \pm 3.2 i$ & $\omega, \delta$ of G14, G16 \\
IA1 & $-0.09 \pm 2.5 i$ & $\omega, \delta$ of $\mathrm{G} 12, \mathrm{G} 13, \mathrm{G} 14, \mathrm{G} 15$ \\
\hline
\end{tabular}

modes corresponding to the nominal operating condition are highlighted. The system has four interarea modes (IA1-IA4) and six local modes (L1-L6). A significant variation can be observed for the local and interarea modes under the operating conditions. At several operating conditions negative damping is observed for L2, L3, and the interarea modes. Participation factor analysis [1] is performed for the local and the interarea modes to find the states participating in the modes. Table II shows the modes and states participating in them.

The local modes L3 and L6 have participation from the states of only one generator and they are absent when corresponding generator is taken out of the service. The other local modes are found to have participation from more than one generator. Hence, they are found to be present even when one of the generators is taken out of the service. Also note that the interarea modes involve synchronous generators located at different locations of the system. The information in the table offers a clue for the likely locations in power system where the oscillations due to an individual mode is visible. For example, the oscillations due to the mode L6 which has participation from the generator G11 located in Area-2 will not be visible in the measurements taken in the other areas. Similarly, the oscillations due to IA2, IA3, and IA4 which have participation from generators in Area-2, Area-3, and Area-4, could not be observed in measurements from Area-1.

In the present system, it is important to improve the damping of local mode L2 and interarea modes to ensure stability under various possible operating conditions. Since L2 has higher participation from the states of generator G2, a PSS at G2 will improve the damping of L2. For interarea mode damping improvement, a supplementary damping controller using the TCSC is proposed.

\section{A. Computation of Residue}

Having selected the actuators, a PSS at G2 and POD in TCSC, the next step is to select feedback signals for both the controllers. Measurements such as active power, reactive power, and current through all transmission lines in the network, and synchronous generator speed are selected as potential feedback signals. For clarity of presentation, the signals are denoted as follows.
1) $P_{p q}, Q_{p q}$, and $I_{p q}$ are active power flow, reactive power flow and current, respectively through transmission line connecting bus \#p and bus \#q.

2) $\omega_{p}$ speed of generator at bus \#p.

\section{B. Controller Design Approach}

In this paper, PSS and POD controllers are designed to validate the proposed signal selection approach using a simple residue-based approach [4]. Let shift in eigenvalue with incremental change in controller gain is given by (3). A controller transfer function $\mathbf{H}(\mathbf{s})$ is obtained such that $\angle\left(H\left(\lambda_{i}\right)\right)=-\angle R_{i}$. When multiple operating conditions are considered, a preliminary controller design is obtained considering average value of $\angle R_{i}$ under various operating conditions and a further tuning of the controller is carried out using robust pole placement approach [23].

The proposed method assumes that feedback signals are readily available to the controller without delay. However, in practical scenario, each signal will be associated with its own time delay. Various controller design methodologies proposed in [27]-[29] could be used to tackle the problem caused by the time delay uncertainties. Since the objective of this paper is to demonstrate signal selection methodology, the simple residue-based controller design method is used in this paper.

\section{Signal Selection}

Fig. 6 shows the histograms for the magnitude and angle of the residue corresponding to IA1 for transfer function between the TCSC reference input and few selected feedback signals. The signal is labeled in the title of each sub-plot. It is evident from the histograms that both the magnitude and angle of the residues have large variation. The degree of variation is different for each signal. The variation is observed for other modes. The selection of feedback signals for PSS and TCSC controllers are made in accordance with the criteria set out in Section III.

\section{A. Feedback Signal for PSS}

The function of the proposed PSS at the generator G9 is to improve the damping of L2. However, both L4 and IA4 have participation from the states of generator G9 which indicates that the PSS will influence the damping of these modes as well. Criteria-3 is crucial in this situation to avoid possible negative effect on L4 and IA4.

Since the main focus is on L2, the location of potential feedback signals is narrowed down to areas around generator G9. Table III lists nine potential feedback signals. The table also contains $\overline{\left|R_{i}^{k}\right|}, \overline{\angle R_{i}^{k}}$, and $\mathbf{P}_{i}^{\mathrm{rel}, k}$ corresponding to modes $i=$ $L 2, L 4$, and IA4. A comparison could be made against $\mathbf{P}_{i}^{\text {rel }, k}$. The associated error ellipses are displayed in Fig. 7.

The best feedback signal according to Criteria-1 is $I_{09-29}$ which has the largest residue magnitude. However, the signal is ranked third in residue variation as evident from the error ellipse and $\operatorname{det}\left(\mathbf{P}_{L 2}^{\mathrm{rel}, k}\right)$. The second best choice according to Criteria-1 is $\omega_{G 9}$. Incidently, it appears to have the least variation of residue as well. The location of open-loop zero 

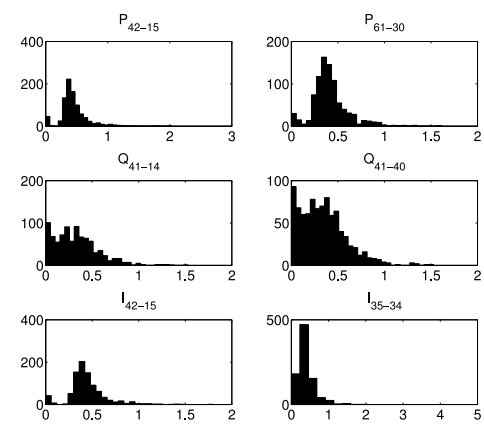

(a)

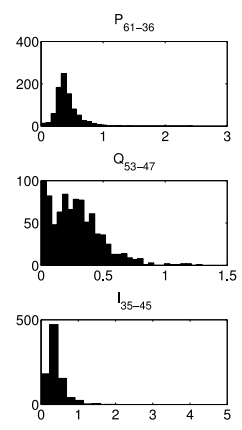

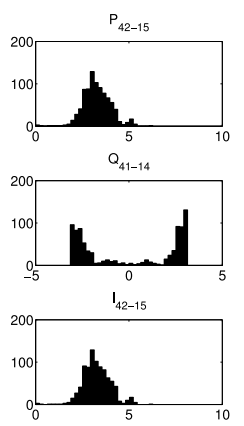

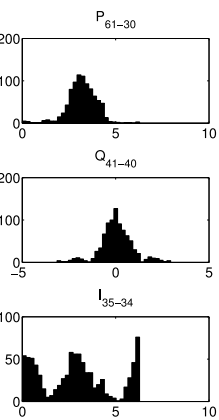

(b)

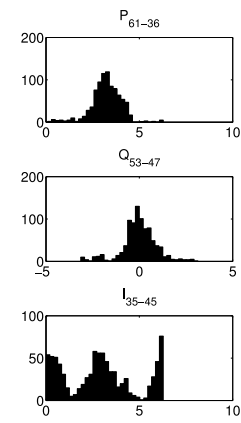

Fig. 6. Histogram showing variation in residue corresponding to IA1 for transfer function between the TCSC reference input and nine feedback signals. The signal name is given as the title of each subplot. (a) Magnitude of residue. (b) Angle of residue.

TABLE III

Characteristics of Possible PSS Feedback Signals

\begin{tabular}{|c|c|c|c|c|c|c|c|c|c|}
\hline $\begin{array}{l}\text { Signal } \\
\text { Name }\end{array}$ & $R_{I}$ & $\begin{array}{c}\operatorname{det}\left(\mathbf{P}_{L 2}^{r e l, k}\right) \\
* 100\end{array}$ & $\overline{R_{I 0}}$ & $R_{\text {}}$ & $\begin{array}{c}\operatorname{det}\left(\mathbf{P}_{I A 4}^{r e l, k}\right) \\
* 100\end{array}$ & $\overline{B_{I}}$ & $\overline{\left|R_{I}\right|}$ & $\begin{array}{c}\operatorname{det}\left(\mathbf{P}_{L 4}^{r e l, k}\right) \\
* 100\end{array}$ & $\overline{\angle R_{L 4}}$ \\
\hline$P_{28-29}$ & $\frac{\mid+L 21}{27.32}$ & 0.0114 & $\frac{21 L 2}{-69}$ & $\begin{array}{c}|+1.05| \\
4.05\end{array}$ & 0.0105 & 81 & $\frac{\mid+24}{7.36}$ & 0.003 & 2 \\
\hline$Q_{28-29}$ & 26.90 & 0.0107 & -67 & 4.07 & 0.0102 & 80 & 7.24 & 0.003 & 0 \\
\hline$I_{28-29}$ & 7.63 & 0.0336 & -67 & 1.46 & 0.1551 & -64 & 2.36 & 0.087 & -140 \\
\hline$P_{26-29}$ & 3.80 & 0.0129 & -46 & 0.93 & 0.1840 & -54 & 1.51 & 0.0007 & -119 \\
\hline$Q_{26-29}$ & 25.15 & 0589 & -89 & 3.45 & & -73 & 6.78 & 60.55 & 180 \\
\hline$I_{26-29}$ & 27.52 & 0441 & -88 & 4.05 & & -72 & 7.42 & 0.048 & -21 \\
\hline$P_{09-29}$ & 7.86 & 0437 & 35 & 9.00 & & -40 & 1.68 & 0.076 & -82 \\
\hline$Q_{09-29}$ & 2.13 & 0.0178 & 60 & 2.39 & 0.0343 & 76 & 0.62 & 0.0045 & -93 \\
\hline$I_{09-29}$ & 54.75 & 0.0440 & -88 & 8.17 & 0.1196 & -72 & 14.76 & 0.0484 & -23 \\
\hline$\omega_{\mathbf{G} 9}$ & 36.51 & 0.0058 & -8 & 8.22 & 0.0096 & -1 & 8.49 & 0.0011 & -82 \\
\hline
\end{tabular}
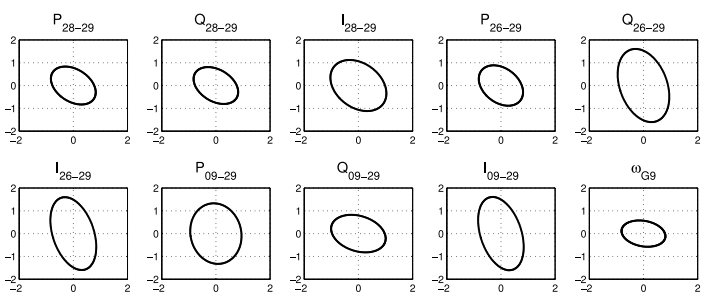

Fig. 7. Error ellipses corresponding to various feedback signals of PSS

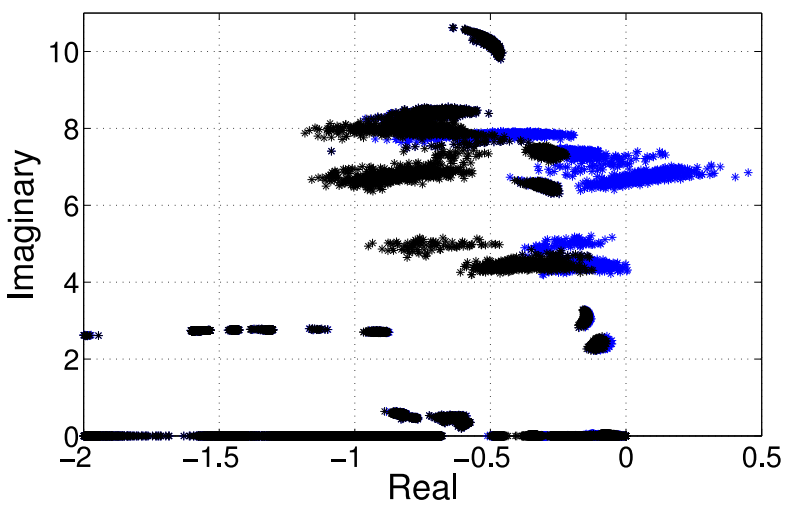

Fig. 8. Eigenvalues of the 16-machine system with and without PSS for all the operating conditions.

showed no adverse effect. Hence, the signal $\omega_{G 9}$ is ideal to improve the damping of mode L2. But it should not have a negative effect on the damping of L4 and IA4. The variation
TABLE IV

Potential FeEdback Signals

\begin{tabular}{c|l|c|c|c|c}
\hline $\begin{array}{c}\text { Signal } \\
\text { Name }\end{array}$ & $\left|R_{I A 1}\right|$ & $\begin{array}{c}\text { det } \\
\left(\mathbf{P}_{I A M 1}^{r e l, k}\right)\end{array}$ & $\begin{array}{c}\text { Signal } \\
\text { Name }\end{array}$ & $\left|R_{I A 1}\right|$ & $\begin{array}{c}\text { det } \\
\left(\mathbf{P}_{I A M 1}^{r e l, k}\right)\end{array}$ \\
\hline$P_{17-13}$ & 1.05 & 0.0189 & $P_{41-14}$ & 0.54 & 0.0299 \\
$P_{42-15}$ & 0.45 & 0.012 & $P_{36-17}$ & 0.75 & 0.0152 \\
$P_{61-30}$ & 0.42 & 0.0118 & $P_{35-34}$ & 0.41 & 0.0239 \\
$P_{34-36}$ & 0.50 & 0.0232 & $P_{61-36}$ & 0.41 & 0.0118 \\
$P_{41-40}$ & 0.46 & 0.012 & $P_{48-40}$ & 0.44 & 0.0127 \\
$P_{18-42}$ & 0.57 & 0.0229 & $P_{35-45}$ & 0.42 & 0.0239 \\
$P_{53-47}$ & 0.52 & 0.0109 & $P_{47-48}$ & 0.49 & 0.011 \\
$P_{45-51}$ & 0.62 & 0.0229 & $P_{50-51}$ & 0.59 & 0.0228 \\
$I_{17-13}$ & 0.94 & 0.0233 & $I_{41-14}$ & 0.58 & 0.0271 \\
$I_{42-15}$ & 0.45 & 0.0104 & $I_{36-17}$ & 0.64 & 0.0535 \\
$I_{35-34}$ & 0.39 & 0.3016 & $I_{34-36}$ & 0.52 & 0.0649 \\
$I_{61-36}$ & 0.39 & 0.0216 & $I_{41-40}$ & 0.36 & 0.0122 \\
$I_{48-40}$ & 0.40 & 0.0226 & $I_{18-42}$ & 0.41 & 0.1235 \\
$I_{35-45}$ & 0.39 & 0.3016 & $I_{47-48}$ & 0.43 & 0.0518 \\
$I_{45-51}$ & 0.54 & 0.0419 & $I_{50-51}$ & 0.64 & 0.0236 \\
\hline
\end{tabular}

in the modal residue corresponding to L4 and IA4 is small. $\overline{\angle R_{L 2}}$ and $\overline{\angle R_{L 4}}$ are very close which indicate a positive contribution to L4 by the PSS using $\omega_{G 9}$ as feedback signal. $\overline{\angle R_{L 2}}$ and $\overline{\angle R_{\mathrm{IA} 4}}$ are in the acceptable range as well. Also note that, $\overline{\left|R_{\mathrm{IA} 4}\right|}$ is $25 \%$ of $\overline{\left|R_{L 2}\right|}$, which suggests that the effect of PSS on IA4 is much less compared to that on L2 and the difference between $\overline{\angle R_{L 2}}$ and $\overline{\angle R_{\mathrm{IA} 4}}$ will not produce appreciably 
TABLE V

Residue Characteristics of Signal $P_{17-13}, P_{61-30}$, And $P_{18-16}$

\begin{tabular}{c||c|c|c||c|c|c||c|c|c}
\hline \multicolumn{1}{l||}{} & \multicolumn{3}{c||}{$P_{17-13}$} & \multicolumn{3}{c||}{$P_{61-30}$} & \multicolumn{3}{c}{$P_{18-16}$} \\
\hline \multirow{2}{*}{ Mode } & $\overline{|R|}$ & $\overline{\angle R}$ & $\operatorname{det}\left(\mathbf{P}^{\text {rel }}\right)$ & $\overline{|R|}$ & $\overline{\angle R}$ & $\operatorname{det}\left(\mathbf{P}^{\text {rel }}\right)$ & $\overline{|R|}$ & $\overline{\angle R}$ & $\operatorname{det}\left(\mathbf{P}^{\text {rel }}\right)$ \\
\hline IA1 & 1.05 & 185 & 0.0189 & 0.42 & 187 & 0.0118 & 0.16 & 206 & 2.0122 \\
IA2 & 8.46 & 101 & 0.0029 & 0.74 & 110 & 0.0360 & 19.30 & 97 & 0.0008 \\
IA3 & 1.96 & -72 & 0.0177 & 0.76 & 117 & 0.0150 & 4.10 & 83 & 0.0156 \\
IA4 & 1.55 & 108 & 0.0954 & 0.53 & 187 & 0.0440 & 0.71 & 177 & 0.8019 \\
\hline
\end{tabular}

negative impact. So $\omega_{G 9}$ is selected as feedback signal for the PSS.

A controller transfer function (4) is obtained for the PSS such that the phase angle of the transfer function at L2 frequency is close to $-\overline{\angle R_{L 2}}$ and the gain is tuned to obtain the required damping for the worst operating condition. Fig. 8 shows the critical modes of the system with and without PSS for all operating conditions. The plot clearly shows the improvement in damping of modes L2, L4, and IA4 while the damping of other modes remain unchanged

$$
\mathbf{K}_{\mathbf{P S S}}=\frac{0.06 s^{2}+0.2 s}{2 s^{2}+10.2 s+1} .
$$

\section{B. Feedback Signal for TCSC}

In order to improve the damping of interarea modes a supplementary controller could be designed using the TCSC [5]. It is decided to find one feedback signal to improve the damping of each interarea mode. In this paper, active power, reactive power, and current of 83 transmission lines along with generator speed from 11 synchronous generators are included in the initial signal set. Altogether 260 potential signals are selected. Although potential signals could have obtained from the corridor only, all locations are considered here for a demonstration purpose.

1) Signal to Improve Damping of IA1: Criteria-1 is applied on the initial set. A set of 30 signals having normalized $\overline{\left|R_{\mathrm{IA} 1}\right|}>0.3$ are identified. The selected signals are listed in Table IV. The table also lists $\overline{\left|R_{\mathrm{IA} 1}\right|}, \overline{\angle R_{\mathrm{IA} 1}}$, and the determinant $\left(\mathbf{P}_{\mathrm{IAM} 1}^{\mathrm{rel}, k}\right)$. Most of the signals listed in the table have less variation in the residue which suggests it passed the first two criteria. The exercise followed the same steps as in the PSS and two cases are described here.

Table V summarizes the residue characteristics of the signal $P_{17-13}$. Although this signal is good for improving damping of IA1, $\overline{\angle R}$ corresponding to other interarea modes are not in favor. Especially $\overline{\angle R_{\mathrm{IA} 3}}$ which is in the other quadrant to $\overline{\angle R_{\mathrm{IA} 1}}$. Considering the magnitude and angle of the residues, the signal comes under Case-C listed in Table I. Hence, this signal is not selected as a feedback signal.

$\mathbf{P}_{61-30}$ is another signal which has passed Criteria-1 and has less variation in residue corresponding to IA1. The residue characteristics are listed in Table V. $\overline{\angle R_{\mathrm{IA} 1}}$ and $\overline{\angle R_{\mathrm{IA} 4}}$ are same whereas the angles of other two modes lie within $90^{\circ}$ which satisfy Criteria-3. $\angle R_{\mathrm{IA} 1}$ varies around $180^{\circ}$ while $\angle R_{\mathrm{IA} 2}$ and $\angle R_{\mathrm{IA} 3}$ are close to $90^{\circ}$ and $\angle R_{\mathrm{IA} 4}$ varies around $180^{\circ}$. The variation in $\angle R_{\mathrm{IA} 2}$ and $\angle R_{\mathrm{IA} 3}$ are less and
TABLE VI

Potential Feedback Signals

\begin{tabular}{l|l|c|c|l|c}
\hline $\begin{array}{c}\text { Signal } \\
\text { Name }\end{array}$ & $\left|R_{I A 2}\right|$ & $\begin{array}{c}\text { det } \\
\left(\mathbf{P}_{I A M 2}^{r e l, k}\right)\end{array}$ & $\begin{array}{c}\text { Signal } \\
\text { Name }\end{array}$ & $\left|R_{I A 2}\right|$ & $\begin{array}{c}\text { det } \\
\left(\mathbf{P}_{I A M 2}^{r e l, k}\right)\end{array}$ \\
\hline$P_{17-13}$ & 8.46 & 0.0029 & $P_{41-14}$ & 11.18 & 0.0009 \\
$P_{18-16}$ & 19.30 & 0.0009 & $P_{35-34}$ & 7.02 & 0.0013 \\
$P_{42-41}$ & 9.05 & 0.0010 & $P_{18-42}$ & 9.02 & 0.0013 \\
$P_{35-45}$ & 7.03 & 0.0013 & $P_{45-51}$ & 9.71 & 0.0013 \\
$P_{50-51}$ & 9.14 & 0.0012 & $I_{17-13}$ & 7.35 & 0.0077 \\
$I_{41-14}$ & 11.40 & 0.0009 & $I_{18-16}$ & 20.99 & 0.0011 \\
$I_{42-41}$ & 6.92 & 0.0340 & $I_{45-51}$ & 7.99 & 0.0314 \\
$I_{50-51}$ & 9.99 & 0.0024 & & & \\
\hline
\end{tabular}

TABLE VII

Potential FeEdback Signals

\begin{tabular}{l|l|c|c|c|c}
\hline $\begin{array}{c}\text { Signal } \\
\text { Name }\end{array}$ & $\left|R_{I A 3}\right|$ & $\begin{array}{c}\text { det } \\
\left(\mathbf{P}_{I A M 3}^{r e l, k}\right)\end{array}$ & $\begin{array}{c}\text { Signal } \\
\text { Name }\end{array}$ & $\left|R_{I A 3}\right|$ & $\begin{array}{c}\text { det } \\
\left(\mathbf{P}_{I A M 3}^{r e l, k}\right.\end{array}$ \\
\hline$P_{41-14}$ & 5.45 & 0.0188 & $P_{42-15}$ & 8.55 & 0.0198 \\
$P_{18-16}$ & 4.10 & 0.0156 & $P_{42-41}$ & 4.82 & 0.0219 \\
$P_{18-42}$ & 3.68 & 0.0203 & $I_{41-14}$ & 5.54 & 0.0187 \\
$I_{42-15}$ & 8.29 & 0.0192 & $I_{18-16}$ & 4.32 & 0.0167 \\
$I_{42-41}$ & 3.68 & 0.0763 & & & \\
\hline
\end{tabular}

the average magnitude of the residue is close to that of IA1. Hence, this signal could be considered as an effective feedback signal. A fixed parameter controller moving IA1 to further left in the eigen-plane will not impact negatively to the damping of other modes.

2) Signal to Improve Damping of IA2: Table VI lists the signals which have passed Criteria-1. All the selected signals have less variation as evident from the determinant of error covariance matrix $(\mathrm{ECM}) . P_{18-16}$ is the signal with least variation in the residue corresponding to IA2 whose characteristics are summarized in Table V. $\angle R_{\mathrm{IA} 2}$ and $\angle R_{\mathrm{IA} 3}$ have less variation and their values are around $90^{\circ} . \angle R_{\mathrm{IA} 1}$ and $\angle R_{\mathrm{IA} 4}$ have higher variation and their values are around $180^{\circ}$. However, the values of $\overline{\left|R_{\mathrm{IA} 1}\right|}$ and $\overline{\left|R_{\mathrm{IA} 4}\right|}$ are significantly less compared to $\overline{\left|R_{\mathrm{IA} 2}\right|}$. Hence, a controller to primarily improve damping of IA 2 will not influence IA1 or IA 4 . Since $\angle R_{\mathrm{IA} 2}$ and $\angle R_{\mathrm{IA} 3}$ are within a narrow range, the direction of shift of IA2 and IA 3 will be almost in the same direction. This makes $P_{18-16}$ a good signal to improve the damping of IA2.

3) Signal to Improve Damping of IA3: Table VII lists the signals which have passed Criteria-1 for IA3. All the selected signals have less variation as evident from the determinant of ECM. The signal $P_{18-16}$ has least variation in the residue 
TABLE VIII

Potential FeEdBack Signals

\begin{tabular}{c|l|c|c|c|c}
\hline $\begin{array}{c}\text { Signal } \\
\text { Name }\end{array}$ & $\overline{\left|R_{I A 4}\right|}$ & $\begin{array}{c}\text { det } \\
\left(\mathbf{P}_{I A M 4}^{r e l, k}\right)\end{array}$ & $\begin{array}{c}\text { Signal } \\
\text { Name }\end{array}$ & $\left|R_{I A 4}\right|$ & $\begin{array}{c}\text { det } \\
\left(\mathbf{P}_{I A M 4}^{r e l, k}\right)\end{array}$ \\
\hline$P_{36-17}$ & 1.61 & 0.0748 & $P_{17-13}$ & 1.55 & 0.0954 \\
$I_{17-13}$ & 1.55 & 0.0805 & $I_{36-17}$ & 1.42 & 0.1436 \\
$I_{61-36}$ & 1.42 & 0.0601 & $P_{61-36}$ & 1.41 & 0.0752 \\
$P_{42-15}$ & 1.40 & 1.2296 & $I_{42-15}$ & 1.35 & 1.2187 \\
$P_{60-61}$ & 0.98 & 0.0576 & $P_{53-54}$ & 0.97 & 0.0506 \\
\hline
\end{tabular}

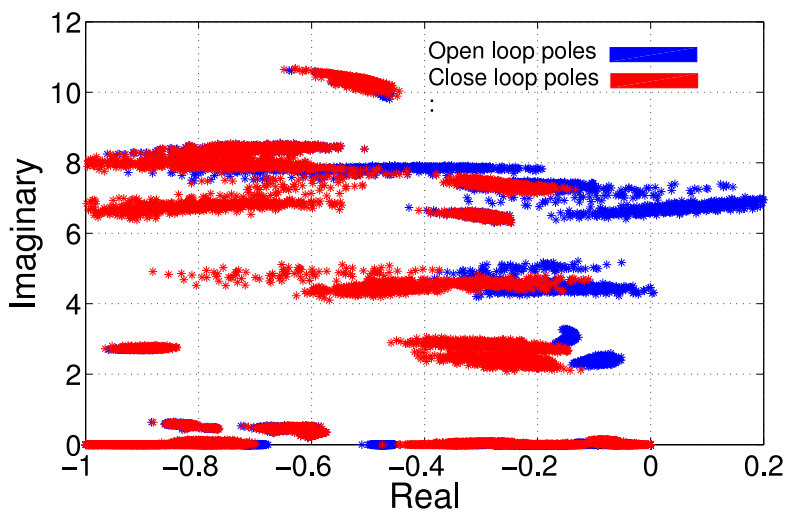

Fig. 9. Electromechanical modes of the 16-machine system without controller, and with PSS and POD.

as evident from determinant of ECM. This is also the signal selected to improve damping IA2 and it is suitable for IA 3 as well.

4) Signal to Improve Damping of IA4: From 260 potential feedback signals, 40 signals passed Criteria-1 for IA4. Few of those signals are listed in Table VIII. However, a significant variation is observed in the residue corresponding to IA4 as evident from higher determinant of ECM. This is also verified using visual inspection of the residue. If the TCSC is used to damp the IA4, it will result in unexpected eigenvalue movement. Hence, it is not advisable to use the TCSC to improve the damping of IA4. Moreover, the PSS can provide sufficient damping for IA4 for stable operation.

Influence of both the selected feedback signals on local modes is also checked to ensure a positive damping action. The controller design and validation is discussed next.

5) Controller Design: A two-input and single-output controller for the TCSC is designed using the selected feedback signals. The controller is designed to improve the damping of IA1, IA2, and IA3 while preserving the damping of other modes.

Table V lists the residue characteristics of both the feedback signals. The signal $P_{61-30}$ with $\overline{\angle R_{\mathrm{IA} 1}}$ equals to $185^{\circ}$ is selected to improve damping of IA1. This angle will decide phase of $\mathbf{K}_{\mathbf{1}}(\mathbf{s})$ (5). Similarly, $P_{18-16}$ is selected for IA2 and IA3 with $\overline{\angle R_{\mathrm{IA} 2}}$ and $\overline{\angle R_{\mathrm{IA} 3}}$ equal to $97.5^{\circ}$ and $83.46^{\circ}$, respectively, which decides phase of $\mathbf{K}_{\mathbf{2}}$ (s)

$$
\mathbf{K}=\left[\begin{array}{l}
\mathbf{K}_{\mathbf{1}} \\
\mathbf{K}_{\mathbf{2}}
\end{array}\right]=\frac{1}{2 s^{2}+10.2 s+1}\left[\begin{array}{c}
-s^{2}-4 s \\
0.0004 s^{2}+0.4 s
\end{array}\right] .
$$

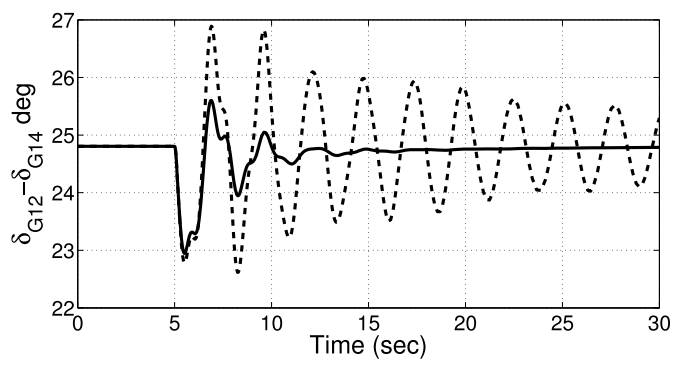

(a)

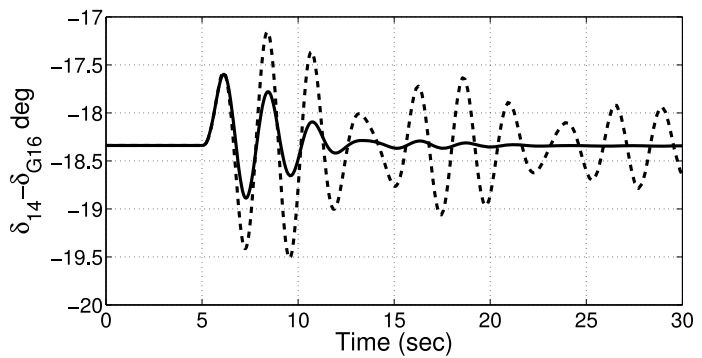

(b)

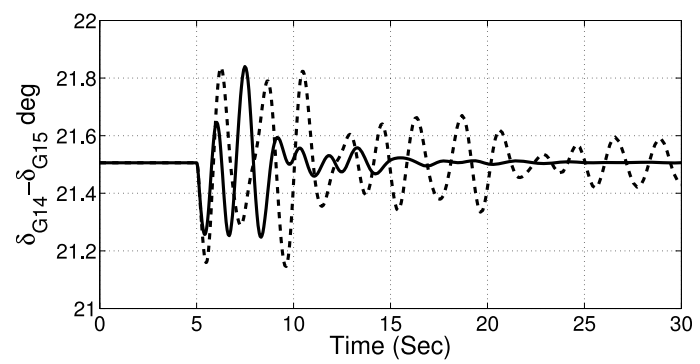

(c)

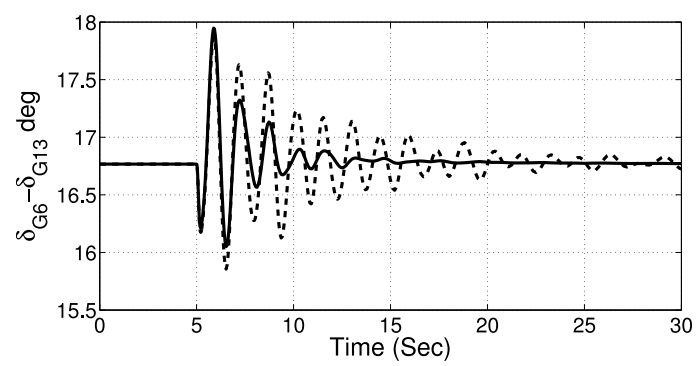

(d)

Fig. 10. Time domain results obtained without controller (dashed lines) and with controller (solid lines). (a) IA1. (b) IA2. (c) IA3. (d) IA4.

Fig. 9 shows the closed loop and open loop poles of the system with and without damping controllers, respectively. The blue shade indicates the open-loop poles for all operating conditions and the red shade displays closed loop poles obtained using the PSS and the TCSC damping controllers. It is clear from the plot that, all the modes have obtained positive improvement in damping ratio. Importantly, the proposed signal selection approach helps to design a controller with very simple structure using simple methods and it inherently ensures robust controller performance over a large number of operating conditions.

In order to validate the feedback signal selection and performance of the controller design, time domain simulation studies 
are performed on the system. A balanced short circuit fault is applied at bus 30 at time $t=5 \mathrm{~s}$. The fault is cleared at the end of three cycles. Fig. 10 shows rotor angle difference between selected synchronous generators in the system with and without using damping controllers. The results are obtained for nominal operating conditions. It is evident from the figures that the controllers offer improved damping for the system oscillatory modes.

\section{CONCLUSION}

This paper presents a set of criteria for feedback signal selection for power system damping controller design. The criteria are based on previously well understood control system tools and suitable for power systems with large variation in operating conditions. This paper sets guidelines for the correct application of the criteria. The feedback signals selected based on the proposed approach allows one to apply simple controller design methods and inherently ensures robust damping for large number of operating conditions. The proposed approach is validated using simulation studies performed on a modified model NETS-NYPS test system with about $12 \%$ wind penetration.

\section{REFERENCES}

[1] P. Kundur, Power System Stability and Control. New York, NY, USA: McGraw-Hill, 1994

[2] L. Fan, H. Yin, and Z. Miao, "On active/reactive power modulation of DFIG-based wind generation for interarea oscillation damping," IEEE Trans. Energy Convers., vol. 26, no. 2, pp. 513-521, Jun. 2011.

[3] L. Rouco and F. Pagola, "An eigenvalue sensitivity approach to location and controller design of controllable series capacitors for damping power system oscillations," IEEE Trans. Power Syst., vol. 12, no. 4, pp. 1660-1666, Nov. 1997.

[4] M. Aboul-Ela, A. Sallam, J. McCalley, and A. Fouad, "Damping controller design for power system oscillations using global signals," IEEE Trans. Power Syst., vol. 11, no. 2, pp. 767-773, May 1996.

[5] B. Chaudhuri and B. Pal, "Robust damping of multiple swing modes employing global stabilizing signals with a TCSC," IEEE Trans. Power Syst., vol. 19, no. 1, pp. 499-506, Feb. 2004.

[6] P. Pourbeik and M. Gibbard, "Simultaneous coordination of power system stabilizers and FACTS device stabilizers in a multimachine power system for enhancing dynamic performance," IEEE Trans. Power Syst., vol. 13, no. 2, pp. 473-479, May 1998.

[7] J. Ferraz, N. Martins, and G. Taranto, "Coordinated stabilizer tuning in large power systems considering multiple operating conditions," in Proc. IEEE Power Eng. Soc. (PES) Gen. Meeting, Tampa, FL, USA, 2007, pp. $1-8$.

[8] N. Martins and L. T. G. Lima, "Determination of suitable locations for power system stabilizersand static VAR compensators for damping electromechanical oscillations in large scale power systems," IEEE Trans. Power Syst., vol. 5, no. 4, pp. 1455-1469, Nov. 1990.

[9] H. Wang, "Selection of robust installing locations and feedback signals of FACTS-based stabilizers in multi-machine power systems," IEEE Trans. Power Syst., vol. 14, no. 2, pp. 569-574, May 1999.

[10] L. Fan and A. Feliachi, "Effective signal selection in decentralized control design of nonlinear interconnected systems," in Proc. 40th IEEE Conf. Decision Control, Orlando, FL, USA, 2001, pp. 3020-3024.

[11] L. Kunjumuhammed, R. Singh, and B. Pal, "Robust signal selection for damping of inter-area oscillations," IET Gener. Transmiss. Distrib., vol. 6, no. 5, pp. 404-416, May 2012.

[12] L. Kunjumuhammed, R. Singh, and B. Pal, "Probability based control signal selection for inter area oscillations damping using TCSC," in Proc. IEEE Power Energy Soc. (PES) Gen. Meeting, Minneapolis, MN, USA, 2010, pp. 1-6.

[13] L. Fan and A. Feliachi, "Damping enhancement by TCSC in the western US power system," in Proc. IEEE Power Eng. Soc. (PES) Winter Meeting, 2002, pp. 550-555.
[14] S. Ray, B. Chaudhuri, and R. Majumder, "Appropriate signal selection for damping multi-modal oscillations using low order controllers," in Proc. IEEE Power Energy Soc. (PES) Gen. Meeting, Pittsburgh, PA, USA, 2008, pp. 1-7.

[15] U. Mhaskar and A. Kulkarni, "Power oscillation damping using FACTS devices: Modal controllability, observability in local signals, and location of transfer function zeros," IEEE Trans. Power Syst., vol. 21, no. 1, pp. 285-294, Feb. 2006.

[16] J. Hauer, "Robust damping controls for large power systems," IEEE Control Syst. Mag., vol. 9, no. 1, pp. 12-18, Jan. 1989.

[17] L. E. Jones and G. Andersson, "Application of modal analysis of zeros to power systems control and stability," Elect. Power Syst. Res., vol. 46, no. 3, pp. 205-211, Sep. 1998.

[18] X. Yang, A. Feliachi, and R. Adapa, "Damping enhancement in the western US power system: A case study," IEEE Trans. Power Syst., vol. 10, no. 3, pp. 1271-1278, Aug. 1995.

[19] M. Farsangi, Y. Song, and K. Lee, "Choice of FACTS device control inputs for damping interarea oscillations," IEEE Trans. Power Syst., vol. 19, no. 2, pp. 1135-1143, May 2004.

[20] A. Heniche and I. Kamwa, "Control loops selection to damp inter-area oscillations of electrical networks," IEEE Trans. Power Syst., vol. 17, no. 2, pp. 378-384, May 2002.

[21] Y. Zhang and A. Bose, "Design of wide-area damping controllers for interarea oscillations," IEEE Trans. Power Syst., vol. 23, no. 3, pp. 1136-1143, Aug. 2008.

[22] R. Singh, B. Pal, and R. Vinter, "Measurement placement in distribution system state estimation," IEEE Trans. Power Syst., vol. 24, no. 2, pp. 668-675, May 2009.

[23] B. Pal and B. Chaudhuri, Robust Control in Power Systems. New York, NY, USA: Springer, 2005.

[24] J. Rueda, D. Colome, and I. Erlich, "Assessment and enhancement of small signal stability considering uncertainties," IEEE Trans. Power Syst., vol. 24, no. 1, pp. 198-207, Feb. 2009.

[25] T.-H. Yeh and L. Wang, "A study on generator capacity for wind turbines under various tower heights and rated wind speeds using Weibull distribution," IEEE Trans. Energy Convers., vol. 23, no. 2, pp. 592-602, Jun. 2008

[26] J. Seguro and T. Lambert, "Modern estimation of the parameters of the Weibull wind speed distribution for wind energy analysis," J. Wind Eng. Ind. Aerodynamics, vol. 85, no. 1, pp. 75-84, 2000.

[27] W. Yao, L. Jiang, J. Wen, Q. Wu, and S. Cheng, "Wide-area damping controller of FACTS devices for inter-area oscillations considering communication time delays," IEEE Trans. Power Syst., vol. 29, no. 1, pp. 318-329, Jan. 2014.

[28] M. Mokhtari, F. Aminifar, D. Nazarpour, and S. Golshannavaz, "Widearea power oscillation damping with a fuzzy controller compensating the continuous communication delays," IEEE Trans. Power Syst., vol. 28, no. 2, pp. 1997-2005, May 2013.

[29] N. Chaudhuri, S. Ray, R. Majumder, and B. Chaudhuri, "A new approach to continuous latency compensation with adaptive phasor power oscillation damping controller (POD)," IEEE Trans. Power Syst., vol. 25, no. 2, pp. 939-946, May 2010.

Linash P. Kunjumuhammed (S'07-M'12) received the B.Tech. degree from Mahatma Gandhi University, Kerala, India; the M.S. degree from the Indian Institute of Technology Madras, Tamil Nadu, India; and the Ph.D. degree from Imperial College London, London, U.K., in 2002, 2006, and 2012, respectively, all in electrical and electronics engineering.

$\mathrm{He}$ has been a Post-Doctoral Research Fellow with Imperial College London, since 2012.

Bikash C. Pal (M'00-SM'02-F'13) received the B.E.E. (Hons.) degree from Jadavpur University, Calcutta, India; the M.E. degree from the Indian Institute of Science, Bangalore, India; and the Ph.D. degree from Imperial College London, London, U.K., in 1990, 1992, and 1999, respectively, all in electrical engineering.

He is currently a Professor with the Department of Electrical and Electronic Engineering, Imperial College London. His current research interests include state estimation and power system dynamics.

Dr. Pal is an Editor-in-Chief of the IEEE TRANSACTIONS ON SUSTAINABLE ENERGY. 